\title{
Injected flavor as a CS in the conditioned aversion preparation
}

\author{
W. P. BELLINGHAM and D. LLOYD \\ Australian National University, Canberra, Australian Capital Territory, Australia
}

\begin{abstract}
Three experiments were conducted to determine the effectiveness of intravenous (IV) flavor injections in the formation of conditioned taste aversions and in the attenuation of neophobia. In Experiment 1, two groups of rats were permitted to drink either a .1\% saccharin solution or tap water followed immediately by IV injections of lithium chloride (LiCl), and two more groups were given IV injections of a $2 \%$ saccharin solution followed immediately by IV injections of either $\mathrm{LiCl}$ or distilled water. Injected flavor did not serve as an effective CS for the conditioning of an aversion to $.1 \%$ saccharin. The second experiment employed a two-bottle procedure to detect attenuation of neophobia using the injected-flavor technique. It was found that, whether saccharin had been injected intravenously (2\%), injected intraperitoneally (2\% IP), or orally consumed (.1\%), neophobia for .5\% saccharin was attenuated equally relative to controls. CS-US intervals were manipulated in the final experiment such that IP injections of $2 \%$ saccharin solution were followed 0-480 min later by IP injections of $\mathrm{LiCl}$. In this case, it was shown that injected flavor ( $2 \%$ saccharin) could act as an effective CS if the US was delayed (optimally about 120 min) and when the test solution was .1\% saccharin. The delay gradient found in Experiment 3 was interpreted as a generalization gradient where optimum conditioning was displayed at the point where the concentration of saccharin circulating in the animal at the time of illness onset most closely matched the concentration of the test solution.
\end{abstract}

Notwithstanding the many parallels between food aversion learning and other forms of learning, certain characteristics of the learned-food-aversion paradigm are sufficiently unusual to have warranted great interest. For instance, a noteworthy feature is the power of the procedure for the conditioning of associations. A single trial is generally all that is required for the conditioning of marked food aversions. However, although this feature is of interest, it is not unique. In sensitive preparations, such as the conditioned emotional response (CER) paradigm, consistent and reliable differences between experimental and control animals can be demonstrated after only a single pairing of conditioned stimulus (CS) and unconditioned stimulus (US).

The second noteworthy feature of food aversion learning is the apparent inability of many stimuli to serve as an effective CS for the prediction of illness. It has been shown (e.g., Garcia \& Koelling, 1966) that rats punished with footshock for drinking water in the presence of light and sound will learn to depress their consumption of water in those circumstances, although rats punished with illness will not. Similarly, rats punished with illness for drinking water with a distinctive taste will learn to depress their consumption of that water, although rats punished with footshock will not. Such examples of CS/US specificity have led to the development of a thesis (Garcia \&

Katy Gillette-Bellingham prepared this paper on the basis of a draft manuscript left by the late William P. Bellingham. Reprint requests should be addressed to Katy Gillette-Bellingham, c/o the Secretary, Department of Psychology, Australian National University, G.P.O. Box 4, Canberra, A.C.T., Australia 2601.
Ervin, 1968) that exteroreceptive cues (e.g., sound) are associable only with external consequences (e.g., footshock) whereas interoceptive cues (e.g., taste) are associable only with internal consequences (e.g., illness). A number of exceptions have been noted to this observation, however (see, e.g., Garcia, Kimeldorf, \& Hunt, 1957; Krane \& Wagner, 1975). There is evidence, also, that birds are able to associate visual cues more readily with illness than with taste cues (Wilcoxon, Dragoin, \& Kral, 1971; but see also Gillette, Martin, \& Bellingham, 1980). It is, furthermore, not clear on what anatomical basis sensory input should be considered as either interoceptive or exteroceptive (Solomon, 1977).

Possibly the most noteworthy feature of food aversion learning is that CS/US associations may be learned over very long delays (although curves showing the effects, on learning, of reinforcement delays in food aversion preparations are similar in function, but not time base, to those obtained in conventional classical conditioning preparations). It has been suggested that the forming of taste associations over long delays is possible only because of the extremely limited amount of information presented over the taste input channel, on the one hand, and the interoceptive sickness input channel, on the other (Revusky, 1971).

Food aversion learning remains of interest not only because its unusual features have prompted a reexamination of learning principles, but also because these features offer unique technical opportunities for the further investigation of learning. In particular, the fact that CS/US associations in flavor aversion learning can be formed over 
long delays not only directs attention to the mechanisms by which this might be achieved, but also offers the procedural advantage of allowing much more opportunity for experimental manipulation and recording during the CS/US interval.

Unfortunately, the CS/US interval is not the only element of the food-aversion-learning preparation that is temporally extended. Presentation of the CS, through the necessary act of eating or drinking a novel food, is necessarily imprecise and protracted. The inelegance of CS presentation also allows the procedural criticism (e.g., Bitterman, 1975) that the taste of a food may linger in the mouth for some time after the initial taste presentation, or that retching during subsequent illness could re-present the original CS cues.

Similar reservations apply to the time course of the US. It is impossible to deliver illness for a brief predetermined period, or even to define the onset and offset of illness as it follows its own time course. The most serious objections in this regard relate to the use of irradiation as a US. Illness following irradiation appears to last for several hours (Smith \& Schaeffer, 1967), and does not seem even to peak until 30-90 min after exposure (Barker \& Smith, 1974; Carroll \& Smith, 1974). This idiosyncrasy can create the illusion of backward conditioning. Lithium chloride appears the least offensive US from this point of view (Barker, Smith, \& Suarez, 1977), although its use only reduces, rather than eliminates, the problem.

It is just as difficult, therefore, to know the point of offset of the US as it is to know the point of offset of the CS. In the case of the US, however, stimulus onset is also a problem. Although it is reasonable to assume that the onset of flavor exposure (i.e., perception of the CS) occurs from the first moment that food is tasted, it is obviously necessary for absorption to take place before sickness can be assumed to have its onset (i.e., perception of the US). Regardless of whether poison is added to what the animal consumes or is injected into the peritoneal cavity, there is an imprecise delay before it takes effect. However, poison takes effect almost immediately if injected intravenously (IV) because it is distributed within seconds to all parts of the body.

Bradley and Mistretta (1971) assumed that IV administration of the CS is perceived as a discrete presentation"Presumably the sodium saccharin passes rapidly through the tongue in a discrete 'pulse'" (p. 188). Certainly this is the impression obtained from Fishberg, Hitzig, and King (1933), who reported that when saccharin is injected into the bloodstream, humans perceive a sweet taste which passes "with great rapidity from the base to the tip of the tongue" (p. 652). Presentation of an IV injected substance is complete within the time taken for the injection (5 to $10 \mathrm{sec}$ ). Delivery of the CS and US by the IV route does not exclude the possibility that the effects of these substances may persist for some time after initial presentation, but it does ensure that no reservoir of flavor will be available in the animal's stomach for re-presentation during subsequent illness, that US onset will follow as closely as possible the administration of poison, and that no reservoir of poison will be available at the injection site to maintain a prolonged US exposure.

Bradley and Mistretta (1971) are known to have used the IV route for the administration of the $C S$ in a foodaversion-learning paradigm. They injected sodium saccharin at various concentrations into the tail veins of rats, followed, within $20 \mathrm{~min}$, by either 100 or $200 \mathrm{R}$ of wholebody irradiation. They were successful in conditioning aversions to the taste of saccharin. Burešova and Bureš (1977) reported the successful conditioning of an aversion to the taste of saccharin administered via the intraperitoneal (IP) route. A possible criticism of Buresova and Bureš's experiment is that they did not use sensitization controls. Scarborough and McLaurin (1961) failed to condition an aversion to an IP-injected CS.

One study is known to have used the IV route for administration of the US (Wise, Yokel, \& De Wit, 1976). Conditioning was successful. The IP route is an extremely common route for the administration of the US and is known to be effective.

The plan of the present research was to confirm and calibrate the effectiveness of the IV route for the administration of CS and US in the taste-aversion-learning paradigm.

\section{GENERAL METHOD}

\section{Subjects}

The animals used in all experiments were outbred male blackhooded Wistar laboratory rats obtained from the Department of Psychology's breeding colony at the Australian National University. They were around 10 weeks of age and weighed around $200 \mathrm{~g}$. They were housed individually for the duration of the experiment and had free access to standard food pellets.

\section{Procedure}

Pretraining. During pretraining, the animals were placed on a schedule that allowed access to water for only $10 \mathrm{~min}$ per day. During the drinking period, water was available in the home cage from either one or two drinking bottles. Pretraining extended for 3 days in the case of the one-bottle procedure and for 6 days in the case of the two-bottle procedure. When pretraining with two bottles, the left-hand bottle was inserted before the right-hand bottle on the 1st, 3 rd, and 5 th days, and vice versa on the remaining days.

Training. Training was carried out on the day after pretraining was completed. Standard aversion training consisted of exposing the animals to a novel flavor, followed, after some interval, by a sublethal dose of poison. One experiment investigated the attenuation of neophobia rather than the conditioning of an aversion and involved the administration of flavor without poison. The flavor used was always saccharin. Lithium chloride was used as a poison in all cases.

The animals were exposed to the flavor of saccharin either by drinking or by injection of a saccharin solution. In the former case, training of the experimental animals was accomplished by substituting saccharin solution for water in the drinking bottle for that day. The concentration of saccharin in the drinking water was $0.1 \%$ 
( $0.1 \mathrm{~g}$ of sodium saccharin for every $100 \mathrm{ml}$ of tap water) in the case of the one-bottle procedure and $0.5 \%$ in the case of the twobottle procedure. When the two-bottle procedure was followed, both bottles contained saccharin solution on the training day.

When the saccharin solution was injected, training was carried out before the normal drinking period. Injection was either IV or IP, and the dose of saccharin was always $2 \%$ solution at $1 \%$ body weight $(2 \mathrm{~g}$ of sodium saccharin for every $100 \mathrm{ml}$ of vehicle, administered at a volume of $1 \mathrm{ml}$ for every $100 \mathrm{~g}$ of body weight). This was the dose used successfully by Buresova and Bures (1977), who injected IP, and very close to the most effective strength demonstrated by Bradley and Mistretta (1971), who injected IV (although Bradley and Mistretta injected at a fixed volume of $1 \mathrm{ml}$ per animal regardless of body weight). The vehicle for injections was either distilled water or physiological saline.

Lithium chloride solution was injected either IP or IV after a variable period following exposure to saccharin. Except where otherwise stated, the solution was $0.3 \mathrm{M}$ concentration, administered at a volume of $1 \%$ body weight. The vehicle, again, was either distilled water or physiological saline.

Except where otherwise stated, both IP injections and IV injections (via a tail vein) were given with a .25 -ga needle. When only a single IV injection was given, a standard syringe was used. When more than one injection was given, a butterfly cannula was implanted in a tail vein and taped to the tail. The cannula was either plugged, when not in use, or connected to a syringe of saline, which was depressed at intervals to keep the line open. The success rate for IV injections varied between $50 \%$ and $90 \%$. Animals that were not successfully injected were discarded on the training day. In the case of all IV injections, the injection site was held until bleeding stopped. The tail was then washed in warm water.

With two exceptions, the procedure described by Barrow (1968) was followed for injections into the tail vein. The first exception was in relation to the point of entry into the vein. Although Barrow suggests entering the vein at the base of the tail, during the course of experiments described below, a higher level of success was obtained by injecting closer to the tip of the tail. (The reason for this is unclear. Perhaps, by reason of being less tightly confined by rigid tissues, the vein is more mobile at the base of the tail than it is at the tip.) The second exception concerned the method of restraint used. Barrow used light anesthesia, although this method appeared to be contraindicated for the study of flavor aversion learning, since some anesthetics may have an aversive effect (Rozin \& Ree, 1972). The method of restraint used in the present research was confinement in a "rat bag" of the type described by Bellingham (1980). A Velcro tab at the narrow end of the bag was secured around the animal's neck; this served to ensure that the animal could neither advance nor retreat. The wide end of the bag was gathered and loosely tied around the base of the tail, completing the animal's immobility and leaving only the tail protruding from one end and the head protruding from the other.

Pretesting. The animals were tested on either the 3rd or 4th day after training. The intervening days were identical to pretraining days, and on the last of these (i.e., the day before test day), each animal's pretest consumption of water was measured as an index of overall fluid consumption independent of any aversive factor.

Testing. In the case of the one-bottle procedure, testing was accomplished by substituting saccharin solution for tap water in the drinking bottle of every rat for that day. In the one-bottle procedure, the test was with $0.1 \%$ saccharin. In the two-bottle procedure, $0.5 \%$ saccharin solution was substituted for unflavored water in one of the two drinking bottles of every rat for that day. The bottle containing saccharin solution was always inserted into the bottle holder on the left-hand side of the cage, and always before the bottle containing tap water. This procedure ensured that every rat tasted the saccharin solution on the test day. Saccharin prefer- ence was expressed in terms of a preference ratio for each rat, that is, the volume of saccharin solution drunk on the test day was expressed as a proportion of the total volume of fluid (saccharin solution plus tap water) drunk on that day. The preference ratio ranges from 0 (for exclusive consumption of tap water) to 1 (for exclusive consumption of saccharin solution).

\section{EXPERIMENT 1}

The aim of this experiment was to determine whether IV injections could function effectively in the flavoraversion paradigm.

\section{Method}

Four groups of rats differed in how they were treated on the training day. They were all tested with the one-bottle procedure.

The first experimental group (DE) drank a $0.1 \%$ saccharin solution for $10 \mathrm{~min}$, followed immediately by an IV injection of a $0.3-\mathrm{M}$ solution of lithium chloride $(\mathrm{LiCl})$ in distilled water at $1 \%$ body weight. The IV-injection experimentals (Group IVE) received an IV injection of a $2 \%$ solution of saccharin in distilled water at $1 \%$ body weight, followed immediately by an $\mathrm{IV}$ injection of $\mathrm{LiCl}$ as per Group DE. The neophobia controls (Group NC) received an IV injection of saccharin identical to that given Group IVE, followed immediately by an IV injection of distilled water at $1 \%$ body weight. The sensitization controls (Group SC) drank tap water for $10 \mathrm{~min}$, followed immediately by an IV injection of $\mathrm{LiCl}$, as per Groups DE and IVE.

\section{Results and Discussion}

The results of Experiment 1 are plotted in Figure 1. The overall one-way analysis of variance was significant $[F(3,28)=22.88, p<.01]$. By Scheffé's test for multiple comparisons (for $p<.01$, the required value of $F$ is 13.71), Group DE consumed significantly less saccharin than Group NC $(F=50.72)$ and Group SC $(F=36.14)$. Group IVE was not significantly different from either Group NC or Group SC.

Apparently, and unexpectedly, IV injection of the US was effective but IV injection of the CS was not. That is, when the animals drank saccharin, an aversion to the



Figure 1. Mean test-day consumption of .1\% saccharin by the experimental groups that either drank a saccharin solution (DE) or had it injected (IVE), by the neophobia controls (NC), and by the sensitization controls (SC). 
test solution was formed, but when they were injected with saccharin, no aversion was demonstrated.

One disturbing feature of the experiment was the apparent discomfort the animals showed to IV injections of distilled water in the NC and SC groups. Neither Bradley and Mistretta (1971) nor Burešova and Bureš (1977) declared what vehicle was used for administration of injections in their experiments. In the present case, distilled water was chosen as the vehicle for saccharin and $\mathrm{LiCl}$ because of the fear that saline might mask the effective perception of saccharin and compete with lithium at the receptor sites. Thus, potentially, saline might interfere with transfer of IV injected saccharin to the test situation or reduce the effectiveness of IV-injected $\mathrm{LiCl}$ to produce its aversive effects. Distilled water was retained as Group NC's US control injection and Group SC's CS control injection for the sake of consistency. However, since distilled water, being hypotonic, passes through the cell membrane into the cells and bursts them, distilled water is not injected in medical practice. Consequently, in Experiments 2 and 3 , distilled water was retained as the vehicle for IV injection of saccharin and $\mathrm{LiCl}$, but physiological saline was used for the CS and US control injections.

\section{EXPERIMENT 2}

Notwithstanding the findings of Bradley and Mistretta (1971) and Burešova and Bureš (1977), Experiment 1 suggests that injected flavor cannot serve as a CS in a flavoraversion paradigm. This being so, it was decided to determine if intravenous exposure to saccharin would attenuate neophobia if the rats had later opportunity to drink saccharin. We expected a positive result because injected saccharin has been reported to produce extinction of saccharin aversions (Baum, Foidart, \& Lapointe, 1974; Brown, Stewart, \& Hall, 1976).

\section{Method}

All injections were given at a volume of $1 \%$ body weight, and the IV saccharin solution was at a strength of $2 \%$. Saline was used for the CS and US control injections, and distilled water was used as a vehicle for the saccharin and $\mathrm{LiCl}$ injections. The two-bottle procedure was used (see the General Method) because of its greater sensitivity to small differences in taste preference and its successful use by Siegel (1974) for detecting attenuation of neophobia. The saccharin solution used for the purposes of the two-bottle test was at the increased strength of $0.5 \%$ in tap water. This was done because rats display only modest neophobia to the previously used concentration of $0.1 \%$ saccharin in tap water (Domjan \& Gillan, 1976). All animals were trained twice. There were five groups of 10 rats each, distinguished as follows by the treatments they received on the training day. The intravenous experimentals (IVE) received an IV injection of saccharin solution on each of the 2 training days. The intraperitoneal experimentals (IPE) received an IP injection of saccharin solution on each of the 2 training days. The saline controls (SAL) received an IV injection of physiological saline on each of the 2 training days. The drinking controls (DC) received the same treatment as Group SAL, except that the normal drinking session on the training day was with the test saccharin solution rather than tap water. The final group was a procedural control (PC). This group received the same treatment as Group SAL, except that a small drop of the saccharin solution was applied to the injection site before the tail was washed. Group PC was added to determine the effectiveness of tail-washing (see the General Method) for the removal of all traces of saccharin after injection. Saccharin can be tasted in very small concentrations, and over the course of experiments animals were frequently observed biting the needle punctures on their tails after injection.

Consumption of saccharin on the test day was expressed for each animal in terms of the previously described preference ratio.

\section{Results and Discussion}

Figure 2 shows that all the groups exposed to IP and IV saccharin (IPE and IVE) exhibited an attenuation of neophobia similar to that of Group DE, which had drunk saccharin prior to the test. The mean preference ratios on the test day were 0.56 for Group IVE, 0.62 for Group IPE, 0.36 for Group SAL, 0.36 for Group PC, and 0.68 for Group DC. Overall one-way analysis of variance was significant $[F(4,45)=6.99, p<.01]$. By Scheffe's test for multiple comparisons (for $p<.01$, the required value of $F$ is 15.08 , and for $p<.05$, the required value of $F$ is 10.32), Groups IVE and IPE combined drank significantly more saccharin than Groups SAL and PCC combined $(F=17.48, p<.01)$ and significantly more saccharin than Group SAL alone $(F=12.01$, $p<.05$ ). Group IPE alone also drank significantly more saccharin than either Group SAL alone $(F=11.53$, $p<.05)$ or Group PC alone $(F=10.92, p<.05)$. There were no significant differences between Groups IVE, IPE, and DC.

Apparently, injected saccharin can act effectively to attenuate neophobia, and hence is sensed and transferred to the consumption of saccharin-adulterated tap water on the test day. However, injected saccharin did not enter into an association with injected $\mathrm{LiCl}$ as demonstrated in Experiment 1. This is a puzzling set of outcomes, since all the ingredients necessary for an association to be formed would seem to be present in Experiment 1's IVE group. The only reasonable explanation would appear to be that the zero delay imposed between the CS and US in Experiment 1 did not allow sufficient time to process the circulating saccharin. Indeed, such a proposition might

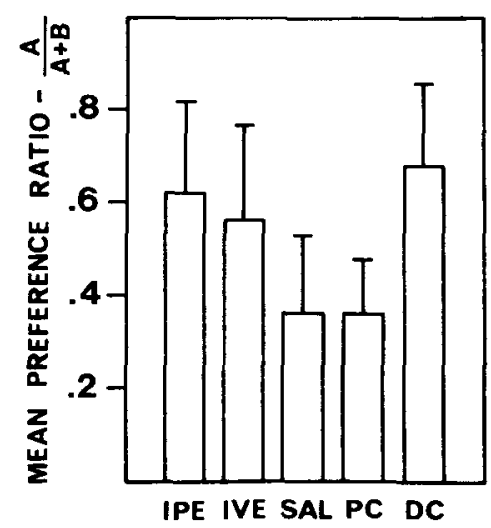

Figure 2. Mean preference ratio for .5\% saccharin of groups given prior injections of 2\% saccharin (IPE and IVE), injections of saline (SAL and PC), or oral presentation of .5\% saccharin (DC). 
very well account for the fact that Bradley and Mistretta (1971) found IV injection of saccharin to be an effective CS, since they used whole-body irradiation to induce illness, a technique known to take considerable time to produce gastrointestinal distress and, therefore, one that would allow more time for injected saccharin to circulate before the onset of illness.

\section{EXPERIMENT 3}

It was decided to vary the interval between injected saccharin and injected $\mathrm{LiCl}$ in order to determine whether a failure to demonstrate an aversion in Experiment 1 could be due to a slow registration of injected saccharin. In the following experiment IP rather than IV injections were used for the sake of technical simplicity.

\section{Method}

There were 10 groups of rats, 8 of which were experimental groups (designated by E) that experienced a CS-US delay of 0,15 , $30,60,120,240,360$, or $480 \mathrm{~min}$.

These rats received an IP injection of saccharin at the beginning of the period and an IP injection of $\mathrm{LiCl}$ at the end of the period.

A further group (Group NC, the neophobia controls) were injected with saline instead of $\mathrm{LiCl}$, and the last group (Group SC, the sensitization controls) were injected with saline instead of saccharin. The CS/US interval for both of the latter two groups was $240 \mathrm{~min}$. Saline was used for control injections, and distilled water was used as a vehicle for $\mathrm{LiCl}$ and saccharin injections. A one-bottle procedure was used. There were 8 animals in each group.

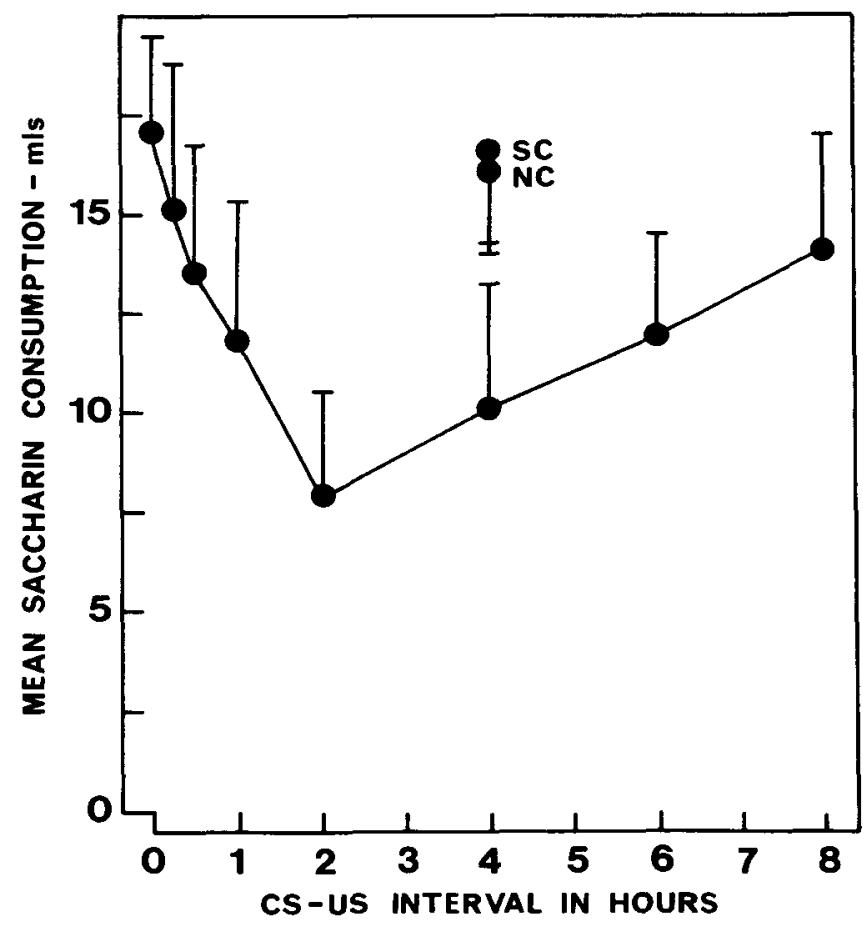

Figure 3. Mean test-day consumption of .1\% saccharin by the experimental groups as a function of CS-US delay, by the sensitization controls (SC), and by the neophobia controls (NC).

\section{Results and Discussion}

Mean consumptions of saccharin solution on the test day are shown in Figure 3. The overall one-way analysis of variance was significant $[F(9,70)=8.85, p<.01]$. Even with the conservative Scheffe analysis, and the large number of groups, there were many significant intergroup differences (for $p<.05$, the required value of $F$ is 18.09). Group E120, for instance, was significantly different from Groups EO, E15, E480, NC, and SC (smallest $F=18.74, p<.05$ ). A trend analysis, excluding Groups NC and SC and using the method of Gaito (1965) for unequal intervals, showed a highly significant quadratic component $[F(1,56)=36.14, p<.01]$.

The empirical fact seems clear: Injected saccharin can act as an effective CS for a taste aversion, but only if some delay exists between the CS and US. The data imply that a delay on the order of $2 \mathrm{~h}$ is optimal for the formation of the association. The result is analogous to that in other preparations in which the optimal CS-US interval is greater than zero. The major, and significant, difference is that the time base in the present instance is on the order of hours rather than seconds.

\section{GENERAL DISCUSSION}

In summary, Experiment 1 demonstrated that IVinjected saccharin was ineffective as a CS when IV LiCl was injected at zero delay. Not surprisingly, when saccharin was externally presented (i.e., drunk), an aversion was demonstrated with a CS-US delay of zero. Experiment 2 demonstrated that IP- and IV-injected saccharin and ingested saccharin were all effective in attenuating neophobia. This allowed for the conclusion that injected saccharin was being sensed and transferred to the testing context, but raised the question of why no association was formed in Experiment 1. The most reasonable explanation seemed to be that injected saccharin, unlike ingested saccharin, needs time to reach a salient level or dissipates less rapidly than injected $\mathrm{LiCl}$. Either interpretation implied that injected saccarin might be an effective CS if the CS-US delay were sufficiently long. Experiment 3 was designed to manipulate the interval between IP-injected saccharin and IP-injected $\mathrm{LiCl}$. It was found that injected saccharin could, indeed, act as an effective CS if the US was delayed, and implied that the optimal CS-US delay was in the vicinity of $2 \mathrm{~h}$.

Bitterman $(1975,1976)$ has argued that the learning of food aversions over long delays may not abrogate the conventional classical conditioning requirement for CS/US contiguity. He suggests that elements of the original taste may persist for some hours until occurrence of the US, and cites Bradley and Mistretta (1971) in apparent support of the proposition that flavor consumption and absorption may lead to subsequent re-presentation of that taste through the intravascular taste mechanism. The findings of this research support his suggestion that intravascular taste perception occurs and may persist for some 
time. The fact that an injected solution of $2 \%$ saccharin may be perceptible for around $2 \mathrm{~h}$ would appear to say little about the likelihood that a consumed solution of $0.1 \%$ saccharin may remain at a perceptible intravascular level for an equivalent period. Notwithstanding the vast difference in saccharin concentrations, however, there is no great difference in terms of the amount of saccharin absorbed by animals in the two circumstances. An animal that weighs $200 \mathrm{~g}$ and drinks $20 \mathrm{ml}$ of a $0.1 \%$ saccharin solution absorbs $20 \mathrm{mg}$ of sodium saccharin, whereas the same animal injected with $2.0 \mathrm{ml}$ of a $2 \%$ saccharin solution absorbs $\mathbf{4 0} \mathrm{mg}$ of sodium saccharin.

The fact that intravascular taste may occur does not, by itself, explain long-delay learning. The point made by Garcia and Hankins (1977) is relevant here. One would scarcely expect an animal to develop light avoidance if the light was turned on and left on several hours before the animal was shocked. Without contesting the essential validity of such a statement, its appropriateness is dependent upon the existence of a one-to-one correspondence between the two procedures. Such is manifestly not the case. Light is a rapid-onset, rapid-offset stimulus, as is shock. Neither taste not $\mathrm{LiCl}$-induced illness display these characteristics. In the analogy, the light is steady state CS. It is unlikely that saccharin, whether ingested normally or injected, can be considered to be in an equivalent steady state. Light is a relatively simple stimulus. Consumption of an adulterated food or liquid must be considered a complex stimulus. Although neither a conditioned-fear procedure nor a conditioned-aversion procedure can be considered a "pure" classical conditioning procedure, the fact that conditioned aversions use similar measures to assess commerce with the CS and CS-US association puts it in a class by itself. There are probably significant differences in the rates of habituation between the visual and taste modalities. In support of the latter statement, Irwin and Bellingham (1980) found that colored water was not an effective CS for chickens poisoned by IP injections of $\mathrm{LiCl}$ at zero delay when the exposure to the colored water was $8 \mathrm{~min}$ or more. However, if exposure was limited to $2 \mathrm{~min}$, an excellent aversion was formed. When the stimulus solution was sodium chloride adulterated water, good aversions were found at exposures of short and long (hours) durations.

One could greatly extend this list at the risk of belaboring the point, namely that the apparently intractable nature of long-delay learning with respect to a generalprocess approach should be taken as evidence of our fundamental ignorance of the important variables that effect associations rather than confirming evidence of the adaptive values of such associations and the superior usefulness of an ethological approach.

Indeed, analytical and ethological approaches are not incompatible, and an analysis of the properties of stimuli that influence association and nonassociation might prove extremely valuable to ethologists by targeting mechanisms and sites of action for long-term genetic adaptation.
It is in this spirit that the following analysis is offered. We subscribe to the view that all stimuli that the organism is capable of sensing are potentially conditionable without leaping inductively to the conclusion that all stimuli are exactly and equivalently conditionable. Different sensory modalities probably vary importantly with respect to properties that determine their tendency to become associated with other sensed events. The previously cited example (Irwin \& Bellingham, 1980) is a case in point, that is, a colored solution is associable with induced illness, but one of its properties, rate of habituation, importantly determines the condition under which that potential is manifested. We also assume that relative contiguity is necessary for associations to occur. Hence, in the case of injected saccharin, the delay gradient found in Experiment 3 is taken to represent a transfer gradient between a training solution of $2 \%$ saccharin injected IP and a test solution of $.1 \%$ saccharin ingested normally when the US is delivered at or near a zero delay. In other words, the delay gradient represents the point at which intravascular circulation of saccharin most closely matches the test solution rather than any true delay gradient. Given shorter or longer circulation times, there is relative transfer (or generalization) decrement. This interpretation assumes that different concentrations of the training or test solutions, different injection routes, or different rates of injection would produce different transfer gradients. However, within a reasonable range of values, all of these variables would be expected to produce a quadratic function.

When saccharin is ingested normally, there is an immediate taste sensation via the taste receptors, as well as a longer term intravascular taste sensation. The immediate stimulation allows an aversion to be formed when the US follows shortly, with intravascular taste producing aversions at the apparently longer delays. In other words, saccharin ingested normally produces both short-term and long-term taste cues. Such a proposition implies that saccharin taste, via receptors on the tongue, should not be able to bridge a long-delay US. Although it has been shown that "external" taste alone is sufficient to produce an aversion (e.g., Domjan \& Wilson, 1972), it has not been shown that taste alone can operate over long delays.

\section{REFERENCES}

BArKer, L. M., \& SMrrH, J. C. (1974). A comparison of taste aversions induced by radiation and lithium chloride in CS-US and US-CS paradigms. Joumal of Comparative \& Physiological Psychology, 87, 644-654.

Barker, L. M., SMrth, J. C., \& SuArez, E. M. (1977). "Sickness" and the backward conditioning of taste aversions. In L. M. Barker, M. R. Best, \& M. Domjan (Eds.), Leaming mechanisms in food selection. Waco, TX: Baylor University Press.

BARrow, M. V. (1968). Modified intravenous injection technique in rats. Laboratory Animal Care, 18, 570-571.

BAUM, M., FoIDART, D. S., a LAPOINTE, A. (1974). Rapid extinction of a conditioned taste aversion following unreinforced intraperitoneal injection of the fluid CS. Physiology \& Behavior, 12, 871-873. 
Belingham, W. P. (1980). An effective, cheap and relatively nontraumatizing rat restrainer. Behavior Research Methods \& Instrumentation, 12, 79-80.

BitTerman, M. E. (1975). The comparative analysis of leaming: Are the laws of learning the same in all animals? Science, 188, 699-709.

Bitterman, M. E. (1976). Reply to Garcia, Hankins, and Rusiniak. Science, 192, 266-267.

Bradley, R. M., Mistretta, C. M. (1971). Intravascular taste in rats as demonstrated by conditioned aversion to sodium saccharin. Joumal of Comparative \& Physiological Psychology, 75, 186-189.

Brown, R. T., STEWART, R. I., \& HALL, T. L. (1976). Extinction of a taste aversion in the absence of the consummatory response. Animal Learning \& Behavior, 4, 213-216.

Burešova, O., Bureš, J. (1977). The effect of anesthesia on acquisition and extinction of conditioned taste aversions. Behavioral Biol$o g y, 20,41-50$.

Carroll, M. E., SMTth, J. C. (1974). Time course of radiationinduced taste aversion conditioning. Physiology \& Behavior, 13, 809-812.

Domun, M., \& Gllan, D. (1976). Role of novelty in the aversion for increasingly concentrated saccharin solutions. Physiology \& Behavior, 16, 537-542.

Doman, M., \& Wison, N. E. (1972). Contribution of ingestive behaviors to taste-aversion learning in the rat. Journal of Comparative \& Physiological Psychology, 80, 403-412.

FishberG, A., Hrtzig, W. M., KING, R. H. (1933). Measurement of the circulation time with saccharin. Proceedings of the Society for Experimental Biology \& Medicine, 30, 651-652.

GarTo, J. (1965). Unequal intervals and unequal $\boldsymbol{n}$ in trend analysis. Psychological Bulletin, 63, 125-127.

Garcia, J., ERVIN, F. R. (1968). Gustatory-visceral and tele-receptorcutaneous conditioning: Adaptation in internal and external milieus. Communications in Behavioral Biology, 1, 389-415.

Garcia, J., \& HankINs, W. G. (1977). On the origin of food aversion paradigms. In L. M. Barker, M. R. Best, \& M. Domjan (Eds.), Learning mechanisms in food selection. Waco, Texas: Baylor University Press.

Garcia, J., Kmeldorf, D. J., Hunt, E. L. (1957). Spatial avoidance behavior in the rat as a result of exposure to ionizing radiation. British Joumal of Radiology, 30, 318-320.

Garcia, J., Koel.nNG, R. A. (1966). Relation of cue to consequence in avoidance learning. Psychonomic Science, 4, 123-124.
Gulette, K., Martin, G. M., Belungham, W. P. (1980). Differential use of food and water cues in the formation of conditioned aversions by domestic chicks (Gallus gallus). Joumal of Experimental Psychology: Animal Behavior Processes, 6, 99-111.

IRWIN, J., \& BelunghaM, W. P. (1980). Differential rates of habituation in single and compound stimuli in young chickens. Australian Psychologist, 15, 267-268.

KRANE, R. V., \& WAGNER, A. R. (1975). Taste aversion learning with a delayed shock US: Implications for the "generality of the laws of learning." Journal of Comparative \& Physiological Psychology, 88, 882-889.

RevUSKY, S. (1971). The role of interference in association over a delay. In W. Honig \& H. James (Eds.), Animal memory (pp. 155-213). New York: Academic Press.

RozIN, P., REE, P. (1972). Long extension of effective CS-US interval by anesthesia between CS and US. Journal of Comparative \& Physiological Psychology, 80, 43-48.

Scarborough, B. B., Mclaurin, W. A. (1961). The effect of intraperitoneal injection on aversive behavior conditioning with $X$ irradiation. Radiation Research, 15, 829-835.

Sizgel, S. (1974). Flavor preexposure and "learned safety." Journal of Comparative \& Physiological Psychology, 87, 1073-1082.

SmTTH, J. C., SChaEFFER, R. W. (1967). Development of water and saccharin preferences after simultaneous exposures to saccharin solution and gamma rays. Joumal of Comparative \& Physiological Psychology, 63, 434-438.

Solomon, R. L. (1977). An opponent-process theory of motivation: V. Affective dynamics of eating. In L. M. Barker, M. R. Best, \& M. Domjan (Eds.), Learning mechanisms in food selection. Waco, TX: Baylor University Press.

Wilcoxon, H. C., Dragoin, W. B., \& Kral, P. A. (1971). Illnessinduced aversions in rat and quail: Relative salience of visual and gustatory cues. Science, 171, 826-828.

WISE, R. A., YOKEL, R. A., \& DEWIT, H. (1976). Both positive reinforcement and conditioned aversion from amphetamine and from apomorphine in rats. Science, 191, 1273-1274.

(Manuscript received May 20, 1985; revision accepted for publication October 29, 1986.) 